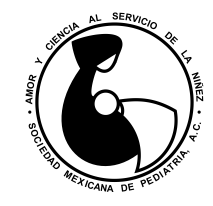

Revista Mexicana de
Vol. 86, No. 4 • Julio-Agosto 2019 pp 151-154

Recibido: 09/10/2018 Aceptado: 07/04/2019

dx.doi.org/10.35366/SP194E

\title{
Encefalitis anti-NMDAR: un caso pediátrico con afasia de expresión
}

\author{
Grecia A Caycho-Gamarra, ${ }^{1, *}$ Mary C Quispe-Rivas, ${ }^{1}$ \\ Nilton Yhuri Carreazo-Pariasca, ${ }^{2}$ Pamela Rocío Muñoz-Huerta ${ }^{3}$ \\ ${ }^{1}$ Universidad Peruana de Ciencias Aplicadas, Sociedad Científica de Estudiantes de Medicina de la Universidad Peruana de \\ Ciencias Aplicadas (SOCIEMUPC); ${ }^{2}$ Universidad Peruana de Ciencias Aplicadas; ${ }^{3}$ Servicio de Neuropediatría del Instituto \\ Nacional de Salud del Niño. Lima, Perú.
}

\begin{abstract}
RESUMEN
Introducción: La encefalitis autoinmune antirreceptor Nmetil-D aspartato (NMDAR) es la causa principal de encefalitis autoinmune aguda en niños y adolescentes, siendo el grupo masculino el que abarca mayor proporción de casos en los menores de 12 años. Presentación del caso: Se presenta el caso de una paciente de seis años de edad que mostró un cuadro clínico sugestivo de encefalitis, siendo lo más resaltante la afasia de expresión, además de hipotonía y crisis convulsivas. Tras descartar una etiología viral, se tomó líquido cefalorraquídeo para la detección de autoanticuerpos NMDAR, la cual fue positiva. Después del inicio de tratamiento con esteroides e inmunoglobulina la paciente mejoró paulatinamente. Conclusiones: La encefalitis autoinmune $\mathrm{N}$-metil-D aspartato debe sospecharse dentro del diagnóstico diferencial de las encefalitis agudas. El tratamiento precoz está relacionado con un mejor pronóstico.
\end{abstract}

Palabras clave: Anticuerpos, encefalitis anti-NMDAR, encefalitis autoinmune, sistema nervioso central.

\section{INTRODUCCIÓN}

La encefalitis es una patología caracterizada por inflamación en el parénquima cerebral que genera una alteración en el funcionamiento neurológico, ${ }^{1}$ cuyas causas pueden ser de origen infeccioso y no infeccioso,

\begin{abstract}
Introduction: Anti-receptor $\mathrm{N}$ methyl-D aspartate (antiNMDA) autoimmune encephalitis is the leading cause of acute autoimmune encephalitis in children and adolescents, with the male group accounting for the highest proportion of cases in children under 12 years of age. Case presentation: We present the case of a female patient of six years of age, who presented signs and symptoms suggestive of encephalitis, the most outstanding were aphasia of expression, hypotonia, seizures and disautonomy. After ruling out a viral etiology, in cerebrospinal fluid sample was performed for the detection of anti-NMDA autoantibodies, which was positive. After the start of steroid and immunoglobulin treatment, the patient gradually improved. Conclusions: Autoimmune N-methyl-D aspartate encephalitis should be suspected as part of the differential diagnosis of acute encephalitis. Early treatment is associated with a better prognosis.
\end{abstract}

Keywords: Antibodies, anti-NMDA encephalitis, autoinmune encephalitis, central nervous system.

siendo la primera la más común; ${ }^{2}$ sin embargo, en los últimos diez años ha aumentado el número de casos de las encefalitis no infecciosas, especialmente las de origen autoinmune. ${ }^{3}$

La encefalitis autoinmune (EA) abarca cuatro grupos de enfermedades: desórdenes paraneoplásicos

\footnotetext{
* Correspondencia: GACG, greciacaycho05@hotmail.com Conflicto de intereses: Los autores declaran que no tienen.

Citar como: Caycho-Gamarra GA, Quispe-Rivas MC, Carreazo-Pariasca NY, Muñoz-Huerta PR. Encefalitis anti-NMDAR: un caso pediátrico con afasia de expresión. Rev Mex Pediatr. 2019; 86(4):151-154.

[Anti-NMDAR encephalitis: a pediatric case with expressive aphasia]
} 
clásicos, enfermedades con autoanticuerpos que atacan proteínas sinápticas intracelulares, desórdenes que presentan autoanticuerpos contra epítopos extracelulares de canales iónicos, receptores y otras proteínas, y otras formas de EA, en las que los antígenos aún no han sido completamente descubiertos. ${ }^{3,4}$

En la EA causada por el antirreceptor $\mathrm{N}$ metilaspartato (NMDAR) se observan anticuerpos contra las subunidades heterómeras NR1 y NR2 de los receptores de glutamato, lo que ocasiona un conjunto de signos y síntomas neuropsiquiátricos característicos de esta enfermedad. ${ }^{5,6}$ Los primeros casos reportados se detectaron en mujeres con teratoma ovárico; sin embargo, posteriormente, el número de casos fue incrementando abarcando ambos sexos, diferentes grupos etarios y sin asociación a la presencia de una neoplasia.,

Un estudio realizado en Reino Unido reveló que 4\% de los pacientes con encefalitis presentaban EA antiNMDAR, siendo más frecuente que otras encefalitis asociadas a anticuerpos. ${ }^{8}$ A continuación se describe el caso de una niña de seis años con el diagnóstico de encefalitis autoinmune anti-NMDAR.

\section{CASO CLÍNICO}

Paciente femenino de seis años de edad, natural y procedente de Lima, Perú, producto de parto eutócico sin complicaciones, con desarrollo psicomotor normal, con esquema de vacunación completa. Como antecedentes patológicos, convulsión febril a los tres años de edad, diarrea disentérica a los cuatro años de edad. Se investigó y descartó contacto con pacientes con tuberculosis.

Acude a emergencia, después de haber sido evaluada en tres centros de salud previamente, por presentar un padecimiento de cuatro días de evolución caracterizado por vómitos postprandiales, dificultad para la deambulación y el habla, así como debilidad muscular en ambos miembros inferiores y un episodio de falta de control de esfínteres. Cabe señalar que no presentó fiebre.

$\mathrm{Al}$ examen físico, se encontraba irritable, afebril, con presión arterial de 90/60 $\mathrm{mmHg}$, taquipnea, con una saturación en $99 \%$ (sin apoyo de oxígeno) y sin adenopatías. En la exploración neurológica: la paciente estaba despierta y parcialmente atenta, nervios craneanos conservados, pupilas isocóricas y reactivas a la luz de 3 a $4 \mathrm{~mm}$, fuerza muscular simétrica $4 / 5$, tono muscular disminuido, ataxia estática a la bipedestación, dismetría en prueba índice nariz, reflejos rotulianos y aquíleos 1+/4+. Signos de Kernig y Brudzinski positivos, afasia de expresión y un puntaje de
11 en la escala de coma de Glasgow (apertura ocular 4 , respuesta verbal 2 , respuesta motora 5).

Los datos de los exámenes de laboratorio fueron los siguientes: hemoglobina $13.2 \mathrm{~g} / \mathrm{dL}, 360,000$ plaquetas $/ \mathrm{mm}^{3}$, 9,600 leucocitos/ $\mathrm{mm}^{3}$ (abastonados $0 \%$ ). Mientras que la glucemia, urea, creatinina, electrolitos, bilirrubinas y proteína $\mathrm{C}$ reactiva se encontraron normales. La tomografía de encéfalo sin contraste no evidenció tumoración, isquemia, hemorragia, ni hidrocefalia.

Se realizó punción lumbar que mostró pleocitosis: 18 leucocitos $/ \mathrm{mm}^{3}$ (78\% mononucleares y $22 \%$ polimorfonucleares), $42.8 \mathrm{mg} / \mathrm{dL}$ de proteínas y $53 \mathrm{mg} / \mathrm{dL}$ de glucosa. La muestra también se envió para buscar posible etiología infecciosa de tipo viral, mediante análisis de reacción en cadena a la polimerasa (PCR viral).

A las 48 horas de haber ingresado, el especialista en Neurología Pediátrica sugirió iniciar pulso de metilprednisolona a $30 / \mathrm{mg} / \mathrm{kg} /$ día por cinco días $(600 \mathrm{mg}$ ) y aciclovir a $20 \mathrm{mg} / \mathrm{kg} /$ dosis (420 mg cada ocho horas), dado que la sospecha diagnóstica era encefalomielitis diseminada aguda (EMDA), o bien, encefalitis viral herpética. Con lo anterior, la paciente mostró mejoría del estado de alerta y ausencia de vómitos; sin embargo, persistió la debilidad muscular e hipotonía, así como signo de Kernig positivo.

La muestra de LCR fue negativa para los 8 virus herpes humanos, poliovirus, echovirus y coxsackievirus. El perfil de coagulación y tiroideo, así como electrolitos, transaminasas y examen de orina fueron normales.

$\mathrm{Al}$ quinto día de hospitalización se suspendió el aciclovir, luego de los resultados negativos del PCR viral. $\mathrm{Al}$ sexto día de hospitalización, mientras se encontraba aún con metilprednisolona en pulso (cuarta dosis), tuvo síntomas compatibles con hipertensión endocraneana (HTE), iniciándose tratamiento para el edema cerebral (manitol al 20\%). Asimismo, hubo hipotermia, catalogada como disautonomía.

La resonancia magnética cerebral de 1.5 teslas con contraste no evidenció tumoración, problema vascular o inflamatoria, incluyendo EMDA, ni eventos isquémicos o hemorrágicos de reciente instalación. El electroencefalograma (EEG) mostró lentificación difusa de trazado con ondas theta $(\theta)$ de 3 a $4 \mathrm{~Hz}$ por segundo.

$\mathrm{Al}$ séptimo día de hospitalización, dado que no había cambio en su condición clínica, se sospechó de una EA con alta posibilidad de encefalitis anti-NMDAR, por lo que se inició inmunoglobulina humana al 5\% a dosis de $2 \mathrm{~g} / \mathrm{kg} / \mathrm{dosis}$ total, repartida en cuatro días (10 gramos cada 24 horas). En esos momentos, tras observarse episodios compatibles con crisis epilépticas 
de tipo focal disperceptivas se inició ácido valproico vía endovenosa a dosis de $20 / \mathrm{mg} / \mathrm{kg} /$ día. Asimismo, luego de terminados los pulsos de metilprednisolona se continuó con prednisona vía oral y se suspendió el manitol al mejorar los signos de HTE. La evolución posterior fue satisfactoria.

Con la finalidad de confirmar el diagnóstico de EA anti-NMDAR, se realizó una segunda punción lumbar que mostró: dos leucocitos $/ \mathrm{mm}^{3}$ (100\% mononucleares), $7 \mathrm{mg} / \mathrm{dL}$ de proteínas, $54 \mathrm{mg} / \mathrm{dL}$ de glucosa, cultivo negativo y no se observaron microorganismos en la tinción Gram. Además, se envió una muestra para la detección de anticuerpos anti-NMDAR a un laboratorio de neuroinmunología, la cual resultó positiva.

La paciente fue dada de alta al mostrar mejoría clínica al día 26 de hospitalización con el diagnóstico de encefalitis autoinmune. En el seguimiento ambulatorio, permaneció asintomática y retornó a las actividades cotidianas propias de su edad.

\section{DISCUSIÓN}

Los desórdenes que presentan autoanticuerpos contra epítopos extracelulares de canales iónicos, receptores y otras proteínas tienen como mejor ejemplo la encefalitis antirreceptor NMDAR, lo que resulta en la disminución del número de receptores sinápticos NMDAR. ${ }^{3,4}$

La EA anti-NMDAR representa la causa principal de EA en niños y adolescentes, con $40 \%$ de casos en pacientes menores de 18 años. ${ }^{8}$ Esta patología predomina en el sexo femenino (81\%) y en jóvenes $(37 \%<18$ años, $95 \%<45$ años); no obstante, esta predilección se invierte en pacientes menores 12 años y mayores 45 años, siendo el grupo masculino el que abarca mayor proporción de casos. ${ }^{9}$ Respecto a la forma paraneoplásica, se ha descrito que representa $40 \%$ de los casos, y la neoplasia que prevalece es el teratoma ovárico en mujeres. ${ }^{7}$ También se han reportado casos en otros tipos de cáncer en varones, como el teratoma testicular y el cáncer de pulmón de células pequeñas. ${ }^{10}$

Los estudios de Lizuka y colaboradores y Sansing y asociados contribuyeron a describir las fases del síndrome de encefalitis autoinmune anti-NMDAR, dentro de las que se incluye la fase prodrómica, psicótica, la fase sin respuesta y, por último, la hipercinética. ${ }^{11,12}$ Respecto a la primera fase, los síntomas de cefalea, fiebre, náuseas, vómitos, diarrea y/o síntomas de tracto respiratorio superior se presentan en aproximadamente $70 \%$ de pacientes. La paciente que aquí describimos mostró rinorrea y tos diez días antes del inicio de su enfermedad, y, al comienzo de la misma, presentó vómitos en varias ocasiones.

En la segunda fase (psicótica), que ocurre aproximadamente dos semanas después de iniciado el cuadro clínico, aparecen otros síntomas dentro de los que destacan ansiedad, manía, miedo, confusión, delusiones de grandeza, paranoia, entre otros. Asimismo, puede presentarse pérdida de memoria a corto plazo; sin embargo, ésta puede ser difícil de evidenciar, pues los síntomas psiquiátricos y los problemas de lenguaje enmascaran este síntoma. ${ }^{13}$ Los problemas de lenguaje son comunes e incluyen una desintegración rápida del mismo, que puede ir desde la reducción del lenguaje, ecolalia y hasta mutismo franco. ${ }^{8}$ Respecto a esto, nuestra paciente mostró una disminución progresiva del lenguaje verbal hasta llegar a la afasia de expresión.

La tercera fase, llamada sin respuesta, se caracteriza por una disminución de la capacidad de responder a estímulos, la que puede fluctuar con periodos de agitación y catatonía. Mientras que, en la última fase, llamada hipercinética, se agregan movimientos anormales como discinesias orolinguofaciales e inestabilidad autonómica, que incluye hipertermia, taquicardia, hipersalivación, hipertensión, bradicardia, hipotensión, como las más frecuentes. ${ }^{8}$ Estos dos últimos signos se observaron en la paciente del caso que presentamos.

El cuadro clínico en niños pequeños puede pasar desapercibido, sobre todo los cambios de comportamiento, como la hiperactividad. En general, el inicio de la sintomatología en niños suele ser de tipo no psiquiátrico, por ejemplo, convulsiones, estatus epiléptico, distonía, reducción verbal o mutismo. ${ }^{14}$ En el caso de nuestra paciente, hubo crisis epilépticas en la evolución clínica durante la hospitalización, además de la afasia expresiva.

El estudio de imagen que suele solicitarse principalmente es la resonancia magnética, ${ }^{15}$ la cual es normal en alrededor de la mitad de los casos. En la otra mitad se puede evidenciar hiperintensidad en la secuencia T2 en algunas zonas cerebrales, como el hipocampo, cerebelo o la corteza cerebral, áreas fronto-basales e insulares, ganglios basales y tronco cerebral. ${ }^{7}$ Asociado a estas características, que suelen ser transitorias o mínimas, se puede observar un leve reforzamiento de contraste en las áreas afectadas o meninges. ${ }^{13} \mathrm{En}$ el caso de la paciente, la resonancia no demostró alteraciones.

Otro de los exámenes que suele apoyar el diagnóstico es el EEG. En los casos de EA anti-NMDAR, este estudio es anormal en aproximadamente $90 \%$ de casos y, por lo regular, muestran un patrón de ralentización generalizada no específica. ${ }^{9}$ Existe un patrón único en 
el EEG denominado complejo delta en cepillo, que se caracteriza por actividad rítmica de $1-3 \mathrm{~Hz}$ con una actividad rítmica superpuesta de $20-30 \mathrm{~Hz}$, que en un estudio se encontró hasta en $30 \%$ de pacientes. ${ }^{16}$ Nuestra paciente registró lentificación difusa de trazado con ondas theta $(\theta)$ de 3 a $4 \mathrm{~Hz}$ por segundo, pero su ritmo era theta $(\theta)$ no delta en cepillo.

Un resultado anormal en LCR se puede detectar hasta en $80 \%$ de los pacientes; las características que deben buscarse incluyen pleocitosis linfocítica, glucosa normal, proteínas elevadas o la presencia de bandas oligoclonales. ${ }^{6,8,13,17}$ Para la confirmación del diagnóstico es necesario demostrar la presencia de autoanticuerpos anti-NMDAR, ya sea en suero o LCR, y es suficiente que uno de ellos salga positivo; ${ }^{19}$ sin embargo, el estándar de oro es el examen en el LCR. ${ }^{8,14,17}$ Los auto-anticuerpos son detectados en ambas muestras simultáneamente hasta en $85 \%$ de casos. ${ }^{13}$ Los resultados del estudio de LCR de la paciente coinciden con los de la literatura.

Dentro del diagnóstico diferencial de esta enfermedad están las causas virales e autoinmunes (encefalitis límbica con autoanticuerpos contra $\mathrm{Hu}, \mathrm{Ma} 2, \mathrm{CV} 2$ y amfifisina). ${ }^{18}$ Asimismo, se debe descartar causas metabólicas y tóxicas. ${ }^{10}$ En el caso de nuestra paciente, fue hasta que se descartaron las causas más frecuentes que se planteó el diagnóstico EA anti-NMDAR y se inició el tratamiento; se conoce que el inicio temprano del tratamiento mejora el pronóstico, lo cual coincide con el caso que presentamos. ${ }^{15}$

El tratamiento de la EA anti-NMDAR consiste en uso de esteroides, inmunoglobulina, plasmaféresis, o una combinación de éstos. En la actualidad, aún no hay evidencia que indique cuál es más efectivo. Lancaster y colaboradores dan preferencia a la inmunoglobulina IV sobre la plasmaféresis en pacientes en edad infantil. ${ }^{19}$ De igual manera, Dalmau y su equipo concuerdan al preferir el tratamiento con inmunoglobulina y metilprednisolona en niños, debido a que la plasmaféresis es un procedimiento más complejo y costoso. ${ }^{8}$ En el caso de no presentar mejoría en un lapso de diez días, se puede cambiar a la segunda línea de tratamiento: rituximab y ciclofosfamida. ${ }^{8}$

\section{CONCLUSIONES}

La EA anti-NMDAR se debe considerar en pacientes que presenten encefalitis de origen desconocido, ya que esta enfermedad es tratable e incluso reversible. El diagnóstico y tratamiento tempranos mejoran el pronóstico de los pacientes.

\section{REFERENCIAS}

1. Tunkel A, Glaser C, Bloch K, Seivar J, Marra C, Roos K et al. The management of encephalitis: clinical practice guidelines by the Infectious Diseases Society of America. Clin Infect Dis. 2008; 47(3): 303-327.

2. Granerod J, Crowcroft NS. The epidemiology of acute encephalitis. Neuropsychol. 2007; 17: 406-428.

3. Leypoldt F, Wandinger K, Bien C, Dalmau J. Autoinmune encephalitis. Eur Neurol Rev. 2013; 8(1): 31-37.

4. Lancaster $\mathrm{E}$. The diagnosis and treatment of autoimmune encephalitis. J Clin Neurol. 2016; 12(1): 1-13.

5. Granerod J, Ambrose H, Daves N, Clewley J, Walsh A, Morgan $D$ et al. Causes of encephalitis and differences in their clinical presentations in England: a multicentre, population-based prospective study. Lancet Infect Dis. 2010; 10(12): 835-844.

6. Gable M, Sheriff H, Dalmau J, Tilley D, Glaser C. The frequency of autoimmune $\mathrm{N}$-methyl-D-aspartate receptor encephalitis surpasses that of individual viral etiologies in young individuals enrolled in the California Encephalitis Project. Clin Infect Dis. 2012; 54(7): 899-904.

7. Dalmau J, Tüzün E, Wu H, Masjuan J, Rossi J, Voloshin A, et al. Paraneoplastic anti-N-methyl-D-aspartate receptor encephalitis associated with ovarian teratoma. Ann Neurol. 2007; 61(1): 25-36.

8. Dalmau J, Lancaster E, Martinez- Hernandez E, Rosenfeld M, Balice- Gordon R. Clinical experience and laboratory investigations in patients with anti-NMDAR encephalitis. Lancet Neurol. 2011; 10(1): 63-74.

9. Titulaer MJ, McCracken L, Gabilondo I, Armangué T, Glaser C, lizuka $T$, et al. Treatment and prognostic factors for long-term outcome in patients with anti-NMDA receptor encephalitis: an observational cohort study. Lancet Neurol. 2013; 12: 157-165.

10. Mooneyham G, Gallentine W, Van Hater H. Evaluation and management of autoimmune encephalitis: a clinical overview for the practicing child psychiatrist. Child Adolesc Psychiatr Clin N Am. 2018; 27(1): 37-52.

11. Lizuka T, Sakai F, Ide T, Monzen T, Yoshii S, ligaya M, et al. AntiNMDA receptor encephalitis in Japan: long-term outcome without tumor removal. Neurology. 2008; 70: 504-511.

12. Sansing LH, Tuzun E, Ko MW, Baccon J, Lynch DR, Dalmau J. A patient with encephalitis associated with NMDA receptor antibodies. Nat Clin Pract Neurol. 2007; 3: 291-296.

13. Dalmau J, Gleichman AJ, Hughes EG, Dalmau J, Gleichman AJ, Hughes EG, Rossi JE, Peng X, Lai M, et al. Anti-NMDA-receptor encephalitis: case series and analysis of the effects of antibodies. Lancet Neurol. 2008; 7: 1091-1098.

14. Florance NR, Davis RL, Lam C, Szperka C, Zhou L, Ahmad S, et al. Anti-N-methyl-D-aspartate receptor (NMDAR) encephalitis in children and adolescents. Ann Neurol. 2009; 66: 11-18.

15. Mann A, Grebenciucova E, Lukas R. Anti-N-methyl-d-aspartatereceptor encephalitis: diagnosis, optimal management, and challenges. Ther Clin Risk Manag. 2014; 10: 517-525.

16. Schmitt SE, Pargeon K, Frechette ES, Hirsch LJ, Dalmau J, Friedman D. Extreme delta brush: a unique EEG pattern in adults with antiNMDA receptor encephalitis. Neurology. 2012; 79(11): 1094-1100.

17. Pruss H, Dalmau J, Harm L, Höltje M, Ahnert-Hilger G, Borowski $\mathrm{K}$, et al. Retrospective analysis of NMDA receptor antibodies in encephalitis of unknown origin. Neurology. 2010; 75: 1735-1739.

18. Wandinger K, Saschenbrecker S, Stoecker W, Dalmau J. AntiNMDA-receptor encephalitis: a severe, multistage, treatable disorder presenting with psychosis. J Neuroimmunol. 2011; 231(1-2): 86-91.

19. Lancaster E, Martinez-Hernandez E, Dalmau J. Encephalitis and antibodies to synaptic and neuronal cell surface proteins. Neurology. 2011; 77(2): 179-189. 